\title{
EDITORIAL OPEN Simplifying a solution to a complex puzzle
}
npj Materials Degradation
(2018)2:36
doi:10.1038/s41529-018-0057-y

Approximately 20,000 tons of borosilicate glass have been produced worldwide so far, for confining high-level radioactive waste generated from the reprocessing of commercial or defense nuclear fuels. The ability of glass structure to incorporate elements throughout the periodic table into a mechanically strong and chemically durable material-with the added convenience of straightforward and mature production processes-makes vitrification the best demonstrated technology for waste immobilization. In particular, the longevity of this material is a critical parameter in limiting the exposure of the biosphere to confined radionuclides, and research has been conducted for decades to study the long-term behavior of these materials and to assess the safety of various disposal concepts. ${ }^{1,2}$

Chemical alteration of glass by groundwater, and to a lesser extent radiation damage to the glass structure, are expected to control the fate of radionuclides confined in glass, determining how long they can be held within the glass structure. ${ }^{1}$ Research has shown that glass durability is not only material dependent, but is also strongly influenced by extrinsic parameters, such as the chemistry of the surrounding solution (such as groundwater), solution renewal rate, temperature, and near field compositions. Although progress has been made in deciphering many of the fundamental processes that control glass corrosion, questions remain regarding the non-linear response of corrosion with glass composition, the role that glass surface layers play in dissolution kinetics, and the evolution of secondary phases over time, to name a few. Yet, all these factors must be understood if successful models are to be built for simulating and predicating the longterm effectiveness of glass for waste immobilization.

In order to provide a common benchmark to relate results generated by different research groups using various experimental tools, including probes from multi-scales, and modeling approaches, it was decided to formulate a common glass that would be studied by all collaborators. ${ }^{3}$ This decision was made during the 4th International Workshop on Glass Corrosion held in Savannah, GA, USA (May 2011). This glass, called the International Simple Glass (ISG), has the following composition (mol\%): $60.2 \mathrm{SiO}_{2}, 16.0 \mathrm{~B}_{2} \mathrm{O}_{3}, 12.6 \mathrm{Na}_{2} \mathrm{O}, 3.8 \mathrm{Al}_{2} \mathrm{O}_{3}, 5.7 \mathrm{CaO}$, and $1.7 \mathrm{ZrO}_{2}$. The main oxide components of this glass are common to most boroaluminosilicate glasses for nuclear waste immobilization, and the elemental ratios are the same as those of SON68 glass (the inactive reference glass of the French R7T7 glass produced by Areva at La Hague), which is arguably the most studied nuclear waste glass to date. To achieve homogeneity between glass samples, a $50 \mathrm{~kg}$ batch of ISG was produced in May 2012 by MoSCI Corporation (Rolla, MO, USA). From May 2012 to May 2018, 55 ingots of $\sim 500 \mathrm{~g}$ (Fig. 1) were distributed to 23 laboratories from seven countries, and $\sim 20$ scientific papers have been published in the literature so far at the time of writing. et al. Historically a similar composition was studied at CEA at the end of the 1990s, providing sound arguments in favor of this glass as a good compromise between simplification and representativeness. ${ }^{4}$
Although 'simple' based on its number of constituents compared to actual nuclear glass composition, this glass is still complex from a fundamental point of view. Six cations means tens of different $\mathrm{A}-\mathrm{O}-\mathrm{A}$ or $\mathrm{A}-\mathrm{O}-\mathrm{B}$ (where $\mathrm{A}$ and $\mathrm{B}$ represent different cations) linkages with variations in bond lengths and bond angles. It is thus not surprising that its structure remains incompletely known and only a combination of spectroscopic and computational modeling approaches can shed light on its short-range and medium-range structural orders. ${ }^{5}$ Additionally, the structure, and properties of ISG, depend on how fast the glass is quenched. ${ }^{6}$ Limiting the reference glass to six cations, however, has enabled the possibility of modeling the glass structure with molecular dynamics. ${ }^{5}$ Such models can be utilized to evaluate potential gel structures, examine diffusion, and determine the relative reactivity of certain structural units. ${ }^{7-9}$

In contact with water, the glass matrix first dissolves by ionexchange and hydrolysis of covalent bonds. The rate of these processes is mostly glass composition, temperature, and $\mathrm{pH}$ dependent. ${ }^{10}$ As corrosion proceeds in a solution with appreciable quantities of glass-sourced ions, amorphous alteration layers are formed from the surface of the corroding glass. ${ }^{11}$ This alteration layer (or layers), can either be formed by precipitation of dissolved species or by in situ reorganization of the Si-rich and Al-rich skeleton left behind from the release of the most mobile species (mainly $\mathrm{B}$ and $\mathrm{Na}$ ), depending on the alteration conditions. ${ }^{12-18}$ In silica-rich solution and $\mathrm{pH}<10$, the alteration layers can act as a transport barrier for key ions, effectively acting as a passivating layer like those on corrosion-resistant metals thereby reducing the glass dissolution rate by several orders of magnitude. Interestingly, alteration layers are not specific to ISG or aluminoborosilicate. A recent review has detailed the commonalties and differences observed between the passivation films formed on silicate glasses, ceramics, and metals, and brought forward ideas that delve deep into the mechanisms of degradation of these materials. ${ }^{19}$ For example, the amorphous nature of the films coating both glasses and ceramics allows the incorporation of many exogenous elements supplied by the environment. ${ }^{20}$ This can affect the structure, properties, thermodynamic stability, and the transport of products and reactants. Although noticed, these effects cannot yet be predicted by existing kinetic models.

Further, although the Ostwald rule of stages predicts these amorphous films will transform into crystalline phases, the timing and processes behind this transition are still unsolved questions. It is known, for instance, that iron or iron corrosion products, ${ }^{21}$ as well as cementitious environments, ${ }^{22}$ or Mg-rich solutions can trigger the formation of secondary phases, which, in turn, can accelerate glass corrosion. ${ }^{23-25}$ The impact of radiation and other decay-related effects on corrosion properties is also poorly understood. Under self-irradiation, silicate glasses undergoes structural modifications, but the impact of these modifications on long-term durability is variable. ${ }^{26}$ The forward rate of dissolution is not affected, but the impact on the alteration layers is less clear.

The mechanisms and kinetics of glass degradation are both complex and non-linear. No individual research group will be able to answer all these demanding questions singlehandedly, and it is thus vital that the community works together. ISG provides a 


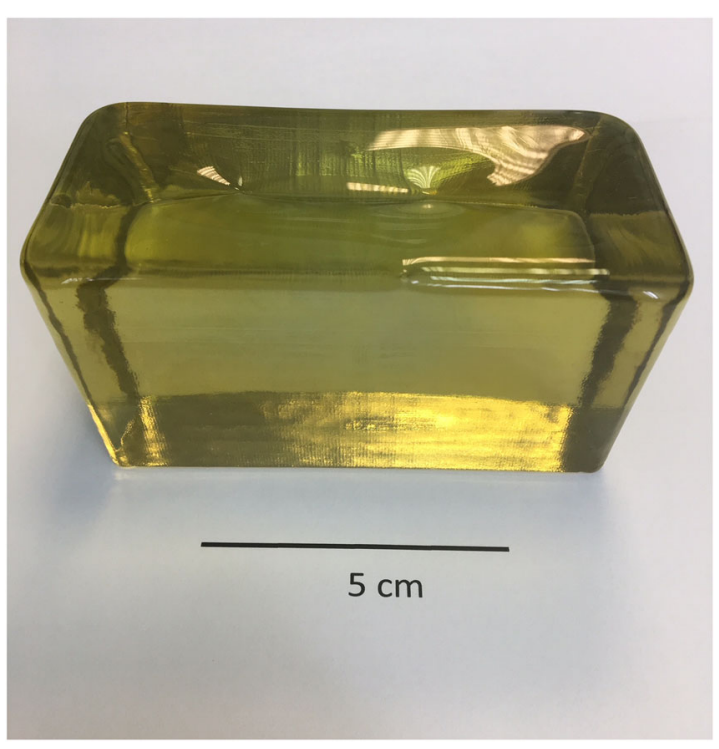

Fig. 1 Ingot of $500 \mathrm{~g}$ of International Simple Glass (ISG) glass produced by $\mathrm{MoSCl}$ corporation

uniform material on which different groups can exert their specific expertize to contribute to a common understanding of glass degradation. Toward this goal, this collection of papers in $n p j$ Materials Degradation on ISG ${ }^{27}$ shows that focusing on a reasonably complex glass is a good way to make progress in the understanding of basic alteration processes. This is a prerequisite for improving predictive models, and for subsequently making informed choices of the best environments for final disposal of vitrified nuclear wastes, as well as the optimal compositions to use. While the puzzle of waste immobilization will not be simple to solve, ISG does provide a common starting point for piecing together understanding.

\section{AUTHOR CONTRIBUTIONS}

S.G., J.R., S.K., and J.D. contributed equally to this editorial.

\section{ADDITIONAL INFORMATION}

Competing interests: The authors declare no competing interests.

Publisher's note: Springer Nature remains neutral with regard to jurisdictional claims in published maps and institutional affiliations.

Stéphane Gin (D), Joseph V. Ryan ${ }^{2}$, Sébastien Kerisit ${ }^{2}$ and Jincheng Du iD $^{3}$

${ }^{1} C E A, D E N$, DE2D, SEVT, F-30207 Bagnols sur Cèze, France; ${ }^{2}$ Pacific Northwest National Laboratory, Physical \& Computational Sciences Directorate, Richland, WA 99352, USA and ${ }^{3}$ Department of Materials Science and Engineering, University of North Texas, Denton, TX, USA Correspondence: Stéphane. Gin (stephane.gin@cea.fr)

\section{REFERENCES}

1. Grambow, B. Nuclear waste glasses-how durable? Elements 2, 357-364 (2006).

2. Gin, S., Jollivet, P., Tribet, M., Peuget, S. \& Schuller, S. Radionuclides containment in nuclear glasses: an overview. Radiochim. Acta 105, 927-959 (2017).

3. Gin, S. et al. An international initiative on long-term behavior of high-level nuclear waste glass. Mater. Today 16, 243-248 (2013).

4. Gin, S., Beaudoux, X., Angeli, F., Jégou, C. \& Godon, N. Effect of composition on the short-term and long-term dissolution rates of ten borosilicate glasses of increasing complexity from 3 to 30 oxides. J. Non-Cryst. Solids 358, 2559-2570 (2012).

5. Collin, M. et al. Structure of International Simple Glass and properties of passivating layer formed in circumneutral $\mathrm{pH}$ conditions. npj Mater. Degrad. 2, 4 (2018).

6. Angeli, F. et al. Effect of thermally induced structural disorder on the chemical durability of International Simple Glass. npj Mater. Degrad. 2, 31 (2018).

7. Du, J. \& Rimza, J. M. Atomistic computer simulations of water interactions and dissolution of inorganic glasses. npj Mater. Degrad. 1, 17 (2017).

8. Lu, X., Deng, L., Kerisit, S. \& Du, J. Structural role of $\mathrm{ZrO}_{2}$ and its impact on properties of boroaluminosilicate nuclear waste glasses. npj Mater. Degrad. 2, 19 (2018).

9. Rimsza, J. M. \& Du, J. Nanoporous silica gel structures and evolution from reactive force field-based molecular dynamics simulations. npj Mater. Degrad. 2, 18 (2018).

10. Vienna, J. D., Neeway, J. J., Ryan, J. V. \& Kerisit, S. N. Impacts of glass composition, $\mathrm{pH}$, and temperature on glass forward dissolution rate. npj Mater. Degrad. 2, 22 (2018).

11. Ngo, D. et al. Spectroscopic ellipsometry study of thickness and porosity of the alteration layer formed on International Simple Glass surface in aqueous corrosion conditions. npj Mater. Degrad. 2, 20 (2018).

12. Gin, S. et al. Dynamics of self-reorganization explains passivation of silicate glasses. Nat. Commun. 9, 2169 (2018).

13. Gin, S. et al. Origin and consequences of silicate glass passivation by surface layers. Nat. Commun. 6, 6360 (2015)

14. Gin, S. et al. The fate of silicon during glass corrosion under alkaline conditions: a mechanistic and kinetic study with the International Simple Glass. Geochim. Cosmochim. Acta 151, 68-85 (2015).

15. Hellmann, R. et al. Nanometre-scale evidence for interfacial dissolutionreprecipitation control of silicate glass corrosion. Nat. Mater. 14, 307-311 (2015).

16. Geisler, T. et al. The mechanism of borosilicate glass corrosion revisited. Geochim. Cosmochim. Acta 158, 112-129 (2015).

17. Lenting, C. et al. Towards a unifying mechanistic model for silicate glass corrosion. npj Mater. Degrad. 2, 28 (2018).

18. Wang, Y., Jove-Colon, C. F., Lenting, C., Icenhower, J. P. \& Kuhlman, K. L. Morphological instability of aqueous dissolution of silicate glasses and minerals. $n p j$ Mater. Degrad. 2, 27 (2018).

19. Frankel, G. S. et al. A comparative review of the aqueous corrosion of glasses, crystalline ceramics, and metals. npj Mater. Degrad. 2, 15 (2018).

20. Collin, M., Fournier, M., Charpentier, T., Moskura, M. \& Gin, S. Impact of alkali on the passivation of silicate glass. npj Mater. Degrad. 2, 16 (2018).

21. Neill, L. et al. Various effects of magnetite on International Simple Glass (ISG) dissolution: implications for the long-term durability of nuclear glasses. npj Mater. Degrad. 1, 1 (2016).

22. Backhouse, D. J. et al. Corrosion of the International Simple Glass under acidic to hyperalkaline conditions. npj Mater. Degrad. 2, 29 (2018).

23. Piovesan, V. et al. Chemical durability of peraluminous glasses for nuclear waste conditioning. npj Mater. Degrad. 2, 7 (2018).

24. Fournier, M., Frugier, P. \& Gin, S. Application of GRAAL model to the resumption of International Simple Glass alteration. npj Mater. Degrad. 2, 21 (2018).

25. Fournier, M., Gin, S., Frugier, P. \& Mercado-Depierre, S. Contribution of zeoliteseeded experiments to the understanding of resumption of glass alteration. $n p j$ Mater. Degrad. 1, 17 (2017).

26. Peuget, S., Tribet, M., Mougnaud, S., Miro, S. \& Jégou, C. Radiations effects in ISGglass: from structural changes to long-term aqueous behavior. npj Mater. Degrad. 2, 23 (2018).

27. International Simple Glass for radioactive waste immobilization. npj Materials Degradation collection https:/www.nature.com/collections/wwcfskftrj (2018).

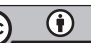

Open Access This article is licensed under a Creative Commons Attribution 4.0 International License, which permits use, sharing, adaptation, distribution and reproduction in any medium or format, as long as you give appropriate credit to the original author(s) and the source, provide a link to the Creative Commons license, and indicate if changes were made. The images or other third party material in this article are included in the article's Creative Commons license, unless indicated otherwise in a credit line to the material. If material is not included in the article's Creative Commons license and your intended use is not permitted by statutory regulation or exceeds the permitted use, you will need to obtain permission directly from the copyright holder. To view a copy of this license, visit http://creativecommons. org/licenses/by/4.0/.

(c) The Author(s) 2018 